\title{
ARTICLE OPEN ReishiMax inhibits mTORC1/2 by activating AMPK and inhibiting IGFR/PI3K/Rheb in tumor cells
}

\author{
Didem Sohretoglu ${ }^{1,2}$, Chao Zhang ${ }^{2,3}$, Jun Luo $^{2,4}$ and Shile Huang (iD $^{2,5}$
}

Ganoderma lucidum (G. lucidum) extracts, as dietary supplements, have been found to exert potent anticancer activity, which is attributed to the presence of polysaccharides and triterpenes. However, the molecular mechanism underlying the anticancer action of $G$. lucidum extracts remains to be investigated. Here, we show that ReishiMax GLp, containing G. lucidum polysaccharides and triterpenes (GLPT), inhibited cell proliferation and induced cell death in human lung cancer cells (A549 and A427) and simultaneously suppressed the signaling pathways of mammalian target of rapamycin complexes 1 and 2 (mTORC1 and mTORC2), respectively. Mechanistically, GLPT downregulated the phosphorylation and protein levels of insulin-like growth factor 1 receptor (IGFR) and phosphoinositide 3-kinase (PI3K) as well as the protein level of RAS homolog enriched in brain (Rheb). In addition, GLPT also activated the AMP-activated protein kinase (AMPK) network. This was evidenced by observations that GLPT increased the phosphorylation of AMPKa (T172) and its substrates tuberous sclerosis complex 2 (TSC2, S1387) and regulatory-associated protein of mTOR (raptor, S792). Ectopic expression of dominant-negative AMPKa partially mitigated the inhibitory effect of GLPT on mTORC1, indicating that GLPT inhibits mTORC1 partly by activating AMPK. The results suggest that G. lucidum extracts exert anticancer action at least partly by suppressing mTORC1/2 signaling via activation of AMPK and inhibition of IGFR/PI3K/Rheb in tumor cells.

Signal Transduction and Targeted Therapy (2019)4:21 ; https://doi.org/10.1038/s41392-019-0056-7

\section{INTRODUCTION}

The medicinal mushroom Ganoderma lucidum (G. lucidum) has been traditionally served as a dietary supplement in Oriental countries to promote health and longevity for a long time. ${ }^{1}$ Evidence has shown that $G$. lucidum exerts a variety of biological activities, including anti-inflammatory, antioxidant, antiglycemic, antiulcer, anticancer, and immunostimulatory effects. ${ }^{1,2}$ Of note, $G$. lucidum executes its anticancer activity mainly via its polysaccharides (from water-soluble extracts) and triterpenes (from waterinsoluble extracts). ${ }^{1,2} \mathrm{G}$. lucidum and its extracts have been documented as potential anticancer agents for various tumors, including those in melanoma, ${ }^{3,4}$ leukemia, lymphoma, myeloma, ${ }^{5,6}$ breast cancer, ${ }^{4-7}$ prostate cancer, ${ }^{4-8}$ ovarian cancer, ${ }^{9}$ bladder cancer, ${ }^{10}$ head and neck cancer, ${ }^{11}$ lung cancer, ${ }^{12-14}$ liver cancer, ${ }^{15,16}$ gastric cancer, ${ }^{17}$ and colon cancer. ${ }^{18,19}$

G. lucidum extracts containing both polysaccharides and triterpenes can directly inhibit cell proliferation, induce cell death and suppress the migration/invasion of tumor cells in vitro and inhibit tumor growth and metastasis in vivo., ${ }^{1,2}$ Studies have reported the various molecular mechanisms underlying these

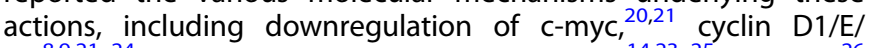
$\mathrm{B} 1{ }^{8,9,21-24}$ cyclin-dependent kinases (CDKs), ${ }^{14,23-25}$ survivin, ${ }^{26}$ vascular endothelial growth factor (VEGF), ${ }^{27,28}$ and matrix metalloproteinase $2 / 9$ (MMP-2/9) ${ }_{i}^{29,30}$ upregulation of CDK inhibitors (p21 $1^{\text {Cip } 1}$ and p2 $\left.7^{\mathrm{Kip} 1}\right) ; 8,22,24$ inhibition of focal adhesion kinase $(\mathrm{FAK}){ }^{31}$ small GTPases, ${ }^{31}$ nuclear factor kappa B (NF-kB), ${ }^{25,32}$ protein kinase $\mathrm{C}$ (PKC), ${ }^{15}$ and $A \mathrm{kt}^{14,33-35}$ and activation of p38 and C-Jun N-terminal kinase (JNK). ${ }^{15,21}$ While it is probable that $G$. lucidum extracts may impact each of these individual signaling molecules depending on the cell types and/or experimental conditions, it seems more conceivable that $G$. lucidum extracts may target certain major targets directly, subsequently influencing the abovementioned targets indirectly.

mTOR (mammalian target of rapamycin) is recognized as a hub that regulates cell growth, survival, and metabolism. ${ }^{36,37}$ Deregulated mTOR signaling has been frequently observed in various types of tumors, so mTOR is regarded as a promising target for cancer therapy. ${ }^{36}$ Current knowledge indicates that mTOR functions as two mTOR complexes (mTORC1 and mTORC2) in mammalian cells. ${ }^{36}$ mTORC1 senses insulin/growth factors, amino acids, energy, oxygen, and DNA damage, while mTORC2 primarily senses insulin/growth factors. ${ }^{36}$ Both mTORC1 and mTORC2 can be positively regulated by the IGFR-PI3K (insulin-like growth factor-1 (IGF-1) receptor-phosphatidylinositol 3' kinase) pathway, which is antagonized by PTEN (phosphatase and tensin homolog). ${ }^{36}$ In addition, mTORC1 is negatively regulated by AMPK

\footnotetext{
${ }^{1}$ Department of Pharmacognosy, Faculty of Pharmacy, Hacettepe University, TR 06100 Ankara, Turkey; ${ }^{2}$ Department of Biochemistry and Molecular Biology, Louisiana State University Health Sciences Center, 1501 Kings Highway, Shreveport, LA 71130-3932, USA; ${ }^{3}$ Key Laboratory of National Health and Family Planning Commission on Parasitic Disease Control and Prevention, Jiangsu Provincial Key Laboratory on Parasite and Vector Control Technology, Jiangsu Institute of Parasitic Diseases, 214064 Wuxi, Jiangsu

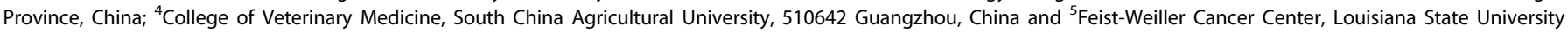
Health Sciences Center, 1501 Kings Highway, Shreveport, LA 71130-3932, USA

Correspondence: Shile Huang (shuan1@lsuhsc.edu)

These authors contributed equally: Didem Sohretoglu, Chao Zhang
}

Received: 10 April 2019 Accepted: 8 May 2019

Published online: 28 June 2019 
(AMP-activated protein kinase). ${ }^{38}$ Low energy levels, oxidative stress or hypoxia activates AMPK, which can phosphorylate TSC2 (tuberous sclerosis complex 2) at multiple sites (including S1387), leading to activation of the TSC $1 / 2$ complex. ${ }^{38,39}$ The activated TSC complex antagonizes Rheb (RAS homolog enriched in brain) by hydrolyzing GTP-Rheb into GDP-Rheb, thereby inhibiting Rhebmediated effects on mTORC $1 .{ }^{39,40}$ In addition, activated AMPK can also phosphorylate regulatory-associated protein of $\mathrm{MTOR}$ (raptor) on S792, leading to inhibition of mTORC1. ${ }^{36}$ While S6K1 (p70 S6 kinase 1) and 4E-BP1 (eukaryotic initiation factor $4 \mathrm{E}$ binding protein 1) are two well-known substrates of mTORC1, Akt (S473) is the best-characterized substrate of $\operatorname{mTORC} 2{ }^{36}$ Although the biological functions of mTORC1/2 remain to be further determined, evidence indicates that mTOR can control the expression/ activity of c-myc, cyclin D1, cyclin-dependent kinases (CDKs), the CDK inhibitor p27 Kip1, VEGF, survivin, JNK, NF-KB, and MMP-2. ${ }^{42}$ Interestingly, of the signaling molecules mediated by mTOR, many of them, e.g., c-myc, cyclin D1, CDKs, p27 ${ }^{\mathrm{Kip} 1}$, survivin, NF-KB, JNK, FAK, small GTPases, MMP-2, and VEGF, are also targeted by $G$. lucidum extracts. ${ }^{20-35}$ Thus, we hypothesized that G. lucidum extracts may exert anticancer effects primarily by targeting mTOR signaling. This study was designed to test this hypothesis using human lung cancer cells (A549 and A427 cells) as experimental models.

\section{RESULTS}

GLPT inhibits cell proliferation and induces cell death in lung cancer cells

It is known that $G$. lucidum executes its antitumor activity primarily via the joint action of triterpenes and polysaccharides [23]. To better evaluate the antitumor activity of G. lucidum, we employed ReishiMax (GLPT), a G. lucidum extract containing $13.5 \%$ polysaccharides and $6 \%$ triterpenes [24]. First, to assess the antiproliferative effect of GLPT on tumor cells, human A549 and A427 lung cancer cells were incubated with GLPT $(0-1 \mathrm{mg} / \mathrm{ml})$ for 3 or 5 days and then enumerated. The results showed that 5 -day treatment with GLPT dose-dependently suppressed cell proliferation (Fig. 1a, b), with $\mathrm{IC}_{50}$ values of $0.39 \mathrm{mg} / \mathrm{ml}$ (A549) and $0.32 \mathrm{mg} / \mathrm{ml}$ (A427). Similar data were obtained in one-solution assays (Fig. 1c, d). These results indicate that GLPT inhibits cancer cell proliferation.

In addition, we also tested the cytotoxic effect of GLPT on A549 and A427 cells. Treatment with GLPT for $72 \mathrm{~h}$ increased the number of trypan blue-positive cells dose-dependently (Fig. 1e). Compared to the vehicle (DMSO), $0.5 \mathrm{mg} / \mathrm{ml} \mathrm{GLPT}$ induced $\sim 4$-fold and 10-fold more cell death in A427 and A549 cells, respectively, whereas $1.0 \mathrm{mg} / \mathrm{ml}$ GLPT was able to increase cell death by nearly 20 -fold in both A427 and A549 cells. Interestingly, at $0.5 \mathrm{mg} / \mathrm{ml}$, GLPT did not exhibit obvious cytotoxic effects on normal (i.e., nontumorous) mouse embryonic fibroblasts (MEFs); at $1.0 \mathrm{mg} / \mathrm{ml}$, GLPT induced massive ( 55\%) cell death in A427 and A549 cells but only induced modest (11\%) cell death in MEFs (Fig. 1e). Collectively, these data suggest that GLPT has potent cytotoxic effects on cancer cells rather than normal cells.

The polysaccharides and triterpenes of $G$. lucidum have been reported to induce apoptosis in a variety of tumor cells. ${ }^{1,2}$ Having observed that treatment with GLPT for $72 \mathrm{~h}$ is able to induce concentration-dependent cell death in A549 and A427 cells (Fig. 1e), we reasoned that GLPT might also induce apoptosis in lung cancer cells. To this end, A427 cells were treated with $0-2 \mathrm{mg} / \mathrm{ml}$ GLPT. After treatment for $72 \mathrm{~h}$, the cells were stained with Annexin V-PI (propidium iodide) and then subjected to flow cytometry, a conventional assay for apoptosis and necrosis. The results showed that treatment with 0.5 and $1.0 \mathrm{mg} / \mathrm{ml} \mathrm{GLPT}$ for $72 \mathrm{~h}$ significantly reduced the percentages of viable cells from $95.2 \%$ (in cells treated with DMSO) to $77.0 \%$ and $45.1 \%$, respectively (Fig. $1 \mathrm{f}, \mathrm{g}$ ). Consistent with this finding, treatment with GLPT increased cell death (apoptosis and necrosis) dose-dependently. GLPT at 0.5 and $1.0 \mathrm{mg} / \mathrm{ml}$ increased the percentages of apoptotic cells from $3.3 \%$ (vehicle control) to $9.4 \%$ and $20.9 \%$, respectively, and increased the percentages of necrotic cells from $1.6 \%$ (vehicle control) to $13.6 \%$ and $34.0 \%$, respectively. The results reveal that GLPT can induce both apoptosis and necrosis in lung cancer cells.

\section{GLPT induces $\mathrm{G}_{1}$ cell cycle arrest in lung cancer cells}

Cell proliferation and cell division are closely associated with cell cycle progression. ${ }^{43}$ To elucidate how GLPT inhibits lung cancer cell proliferation, cell cycle analysis was conducted. As the doubling time of the A549 cell line is $\sim 22 \mathrm{~h}$ (ATCC information sheet), the cells were treated with GLPT for $24 \mathrm{~h}$. We found that such treatment increased the cell population in the $G_{0} / G_{1}$ phase in a dose-dependent manner. GLPT at $0.25,0.5$ and $1.0 \mathrm{mg} / \mathrm{ml}$ significantly increased the proportion of cells in $G_{0} / G_{1}$ phase from $51.3 \%$ (control) to $65.6 \%, 76.1 \%$ and $87.2 \%$, respectively (Fig. $2 a, b$ ). These data suggest that GLPT inhibits lung cancer cell proliferation by inducing $G_{1}$ cell cycle arrest.

Cell cycle progression is tightly regulated by the coordinated activities of cyclin/CDK complexes, CDK inhibitors and Cdc $25 .{ }^{43}$ To elucidate how GLPT induces $G_{1}$ cell cycle arrest in lung cancer cells, we determined the levels of proteins related to $G_{1}$ phase-to$S$ phase progression. Our western blot analysis showed that GLPT treatment $(24 \mathrm{~h})$ dose-dependently reduced the cellular protein levels of cyclin D1, CDK4, CDK6, and Cdc25A and increased the protein levels of the CDK inhibitors $\mathrm{p} 21^{\mathrm{Cip} 1}$ and $\mathrm{p} 27^{\mathrm{Kip} 1}$ (Fig. 2c). Notably, CDK1 and CDK2 were not obviously affected by GLPT treatment, despite a slight inhibitory effect of $2 \mathrm{mg} / \mathrm{ml}$ GLPT on cyclin A/E expression in A549 cells (Fig. 2c). Rb (retinoblastoma protein) acts as a checkpoint protein regulating cell cycle progression from $G_{1}$ phase to $S$ phase. ${ }^{43}$ As predicted, GLPT treatment for $24 \mathrm{~h}$ also inhibited $\mathrm{Rb}$ phosphorylation dosedependently (Fig. 2c). These observations demonstrate that GLPT induces $G_{1}$ cell cycle arrest associated with inhibition of $G_{1}$ cyclins/ $\mathrm{CDKs}$, leading to decreased phosphorylation of $\mathrm{Rb}$.

GLPT induces caspase-dependent apoptosis in lung cancer cells Caspase-dependent and caspase-independent apoptosis can be induced under different conditions. ${ }^{44}$ Having observed GLPTinduced apoptosis in lung cancer cells (Fig. If, g), we wondered whether this apoptosis was caspase-dependent. To answer this question, cleaved PARP, which is a hallmark of caspase-dependent apoptosis, ${ }^{44}$ was probed by western blotting. The results showed that 24-h treatment with GLPT increased the level of cleaved PARP dose-dependently in both A549 and A427 cells (Fig. 3a), indicating caspase-dependent apoptosis. This finding was further supported by the observation that GLPT activated caspase 3, as judged by increased cleavage of caspase 3 in A549 and A427 cells revealed by western blot analysis (Fig. 3a).

Caspase-dependent apoptosis can be mediated by the mitochondrial and death receptor pathways. ${ }^{44,45}$ To determine the pathways involved in the observed apoptosis, we first investigated whether GLPT-induced apoptosis occurs through the mitochondrial pathway. Since the $\mathrm{BCl}-2$ family proteins are the key players in this pathway, the levels of these proteins were tested. We found that treatment with GLPT $(0-2 \mathrm{mg} / \mathrm{ml})$ for $24 \mathrm{~h}$ did not apparently affect the levels of the proapoptotic proteins BAD and BAK, despite a marginal increase in BAX in A549 and A427 cells. In contrast, GLPT treatment greatly decreased the levels of the antiapoptotic proteins survivin and $\mathrm{BCl}-\mathrm{xL}$ in a dose-dependent manner (Fig. 3a). At $2 \mathrm{mg} / \mathrm{ml}$, GLPT also reduced the level of Bcl-2. The results indicate that GLPT-induced apoptosis is partly mediated by activation of the mitochondrial pathway mainly associated with downregulation of survivin, $\mathrm{BCl}-\mathrm{xL}$ and $\mathrm{Bcl}-2$ (antiapoptotic proteins).

Next, we further studied whether the death receptor pathway is involved in GLPT-induced apoptosis. Since caspase 8 is a crucial 
a

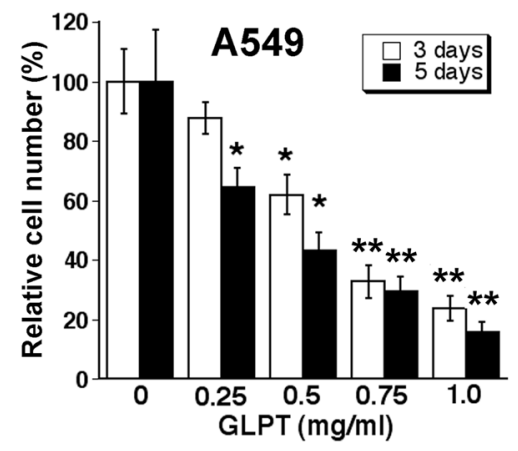

C

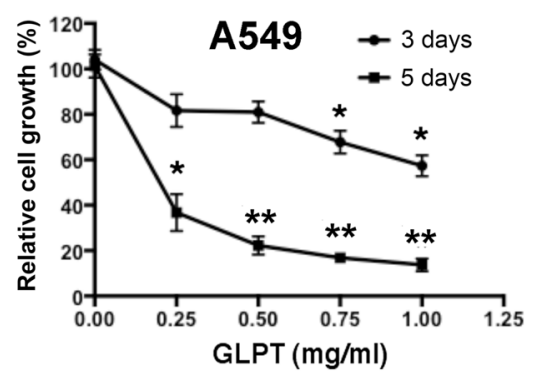

b

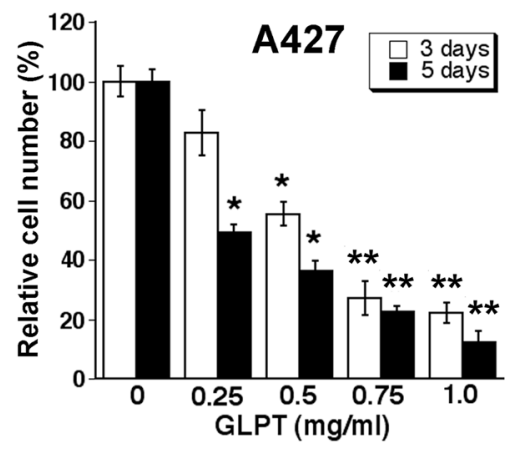

d

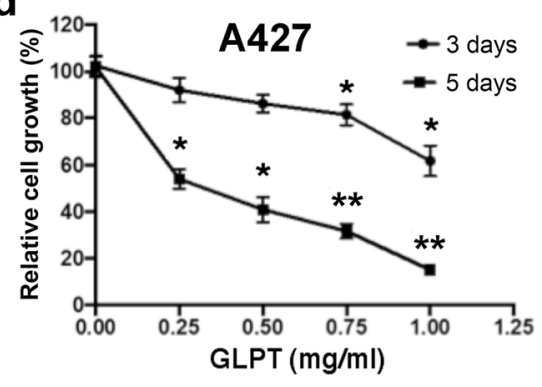

e

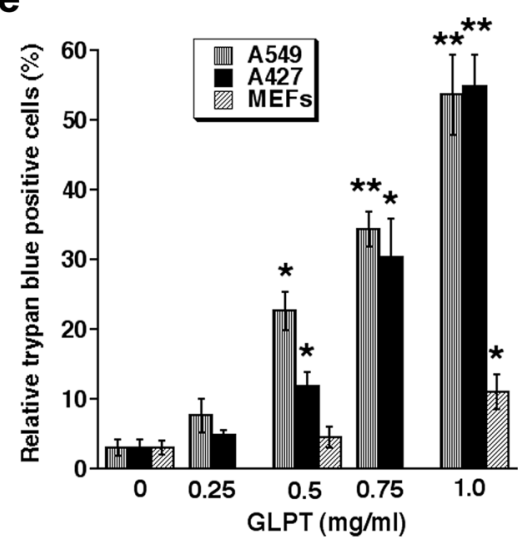

g

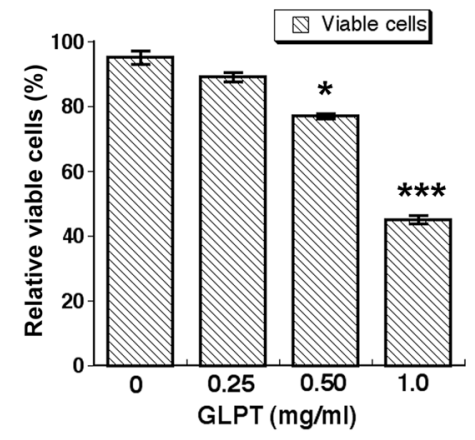

f
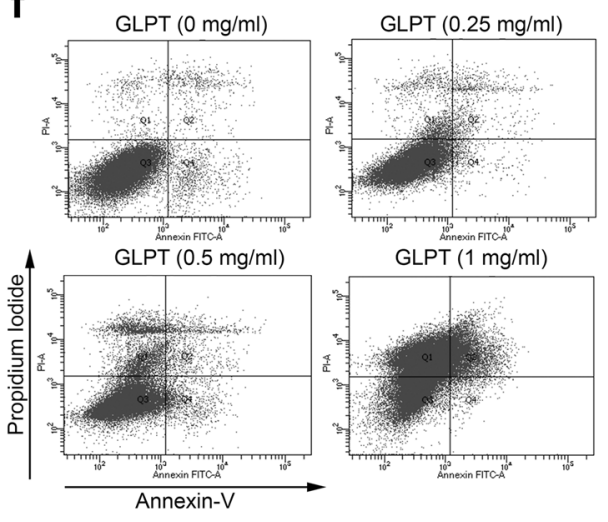

GLPT (1 mg/ml)
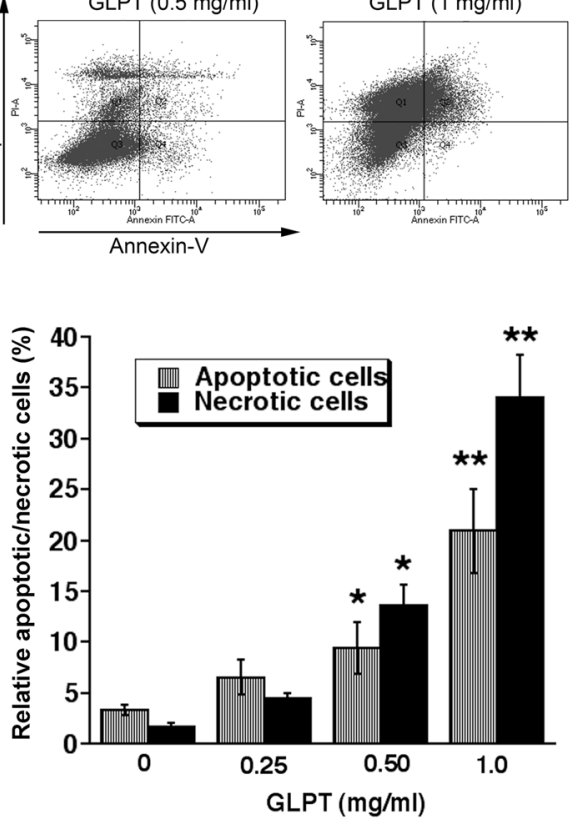

Fig. 1 GLPT inhibits cell proliferation and induces cell death in lung cancer cells. a-d A549 and A427 cells were treated with GLPT (0-1 mg/ml) for 3 or 5 days and then subjected to cell counting $(\mathbf{a}, \mathbf{b})$ or one-solution assay $(\mathbf{c}, \mathbf{d})$. e- $\mathbf{- g}$ The indicated cells were treated with GLPT (0-1 mg/ $\mathrm{ml}$ ) for $72 \mathrm{~h}$ and then analyzed with trypan blue exclusion assay (e) or with annexin V-PI staining followed by flow cytometry (f, $\mathbf{g}$ ). Representative images of the flow cytometry results are shown in (f), and the quantitative data are presented in (g). All data represent the mean \pm SD $(n=3)$. GLPT treatment versus control $(0 \mathrm{mg} / \mathrm{ml}$ GLPT $),{ }^{*} P<0.05 ;{ }^{* *} P<0.01 ;{ }^{* *} P<0.001$ 
initiator caspase for the activation of the death receptor pathway, ${ }^{45}$ we first examined whether GLPT activates caspase 8. Interestingly, treatment with GLPT $(0-2 \mathrm{mg} / \mathrm{ml})$ for $24 \mathrm{~h}$ increased the level of cleaved caspase 8 (Fig. $3 \mathrm{~b}$ ), indicating activation of caspase 8.

To better understand how the death receptor pathway was activated, we investigated whether GLPT affects the levels of related ligands, death receptors, and adaptor proteins. Treatment with GLPT $(0-2 \mathrm{mg} / \mathrm{ml})$ for $24 \mathrm{~h}$ did not obviously influence the expression of TRAIL, TRADD, FASL, FADD, and RIP1 but drastically upregulated the protein levels of DR4 and DR5 (Fig. 3b). Surprisingly, the protein level of TNFR1 was profoundly reduced by GLPT in a dose-dependent manner. In addition, in response to GLPT treatment, RIP3, which is involved in the regulation of necroptosis, ${ }^{46}$ was markedly upregulated in A549 cells but downregulated in A427 cells. The above results suggest that GLPT-induced apoptosis is also in part linked to increased expression of DR4 and DR5 in lung cancer cells. Taken together, our findings reveal that GLPT is able to induce caspase-dependent apoptosis through both the mitochondrial and death receptor pathways.

GLPT inhibits both the mTORC1 and mTORC2 pathways mTOR plays a central role in the regulation of cell proliferation and survival. ${ }^{36}$ To determine whether $G$. lucidum might execute its anticancer action by inhibiting the mTOR pathway, A549 and A427 cells were treated with GLPT $(0-2 \mathrm{mg} / \mathrm{ml})$ for $24 \mathrm{~h}$, and then western blot analysis of mTOR signaling molecules was performed. We observed that GLPT dose-dependently inhibited the phosphorylation of S6K1 (T389), a well-known substrate of mTORC $1,{ }^{36}$ and remarkable inhibition started at $0.5 \mathrm{mg} / \mathrm{ml}$ (Fig. 4a). Similarly, GLPT also inhibited the phosphorylation of 4E-BP1, another major substrate of $\mathrm{mTORC}_{1}{ }^{36}$ in the cells. In addition, treatment with GLPT for $24 \mathrm{~h}$ also dose-dependently decreased the phosphorylation of Akt (S473) (Fig. 4b), a substrate of mTORC2. ${ }^{36}$ Similarly, GLPT also inhibited the phosphorylation of Akt (T308) (Fig. 4b), which is mediated by PDK1 (phosphoinositidedependent kinase 1). ${ }^{36}$ One control, rapamycin, an allosteric inhibitor of mTORC1, inhibited mTORC1-mediated phosphorylation of S6K1 (T389) but increased mTORC2-mediated phosphorylation of Akt (S473) (Fig. 4C); another control, AZD8055, an ATP-competitive and selective inhibitor of mTOR, inhibited both mTORC1 and mTORC2 (Fig. 4c). Collectively, the results indicate that GLPT inhibits both the mTORC1 and mTORC2 signaling pathways in lung cancer cells.

GLPT inhibition of mTORC1/2 is related to activation of AMPK and inhibition of IGFR/PI3K/Rheb

Since both mTORC1 and mTORC2 are positively mediated by the IGFR-PI3K pathway and negatively regulated by PTEN, ${ }^{36,42}$ to understand how GLPT inhibits mTORC1/2, we next investigated whether GLPT inhibits mTORC $1 / 2$ by altering the expression/ activity of these signaling proteins. As predicted, treatment with GLPT for $24 \mathrm{~h}$ dose-dependently reduced the levels of $\mathrm{p}$-IGFR $\beta$, p-PI3K (p85), and p-PDK1 in A549 cells (Fig. 5a). Additionally, GLPT dose-dependently downregulated the protein level of IGFR $\beta$ but did not reduce the protein levels of PI3K (p85) and PDK1 except at concentrations of $1 \mathrm{mg} / \mathrm{ml}$ and higher. Unexpectedly, GLPT treatment inhibited rather than activated PTEN, as indicated by the observation that GLPT decreased the protein level and increased the phosphorylation level of PTEN (Fig. 5a). Thus, the results suggest that GLPT inhibition of $m$ TORC $1 / 2$ is related to downregulation of the IGFR-PI3K pathway but not to upregulation of PTEN.

As mTORC1 is also negatively regulated by the AMPK-TSC2/ raptor network and positively regulated by Rheb, ${ }^{38-41}$ we also examined whether GLPT suppresses mTORC 1 by activating AMPK and inhibiting Rheb. As expected, 24-h treatment with GLPT dose-
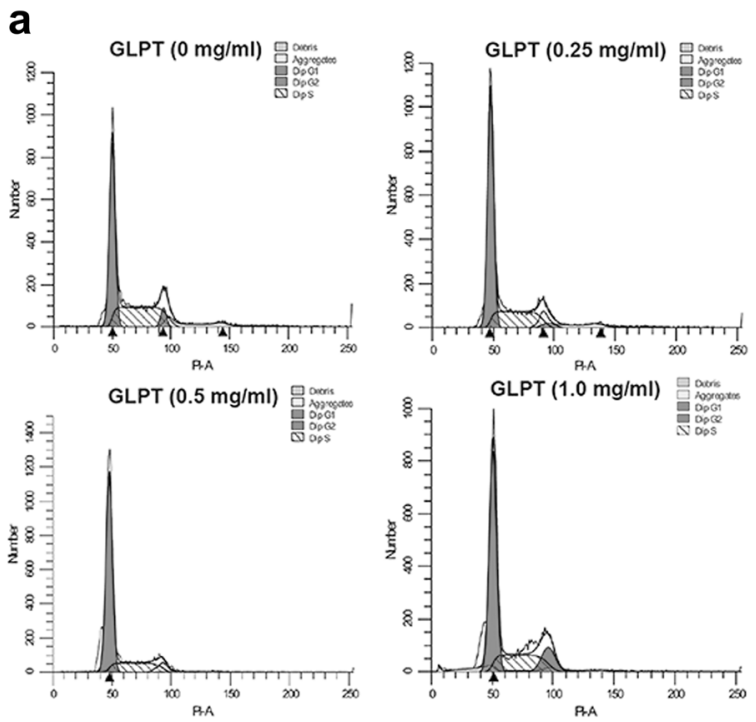

b

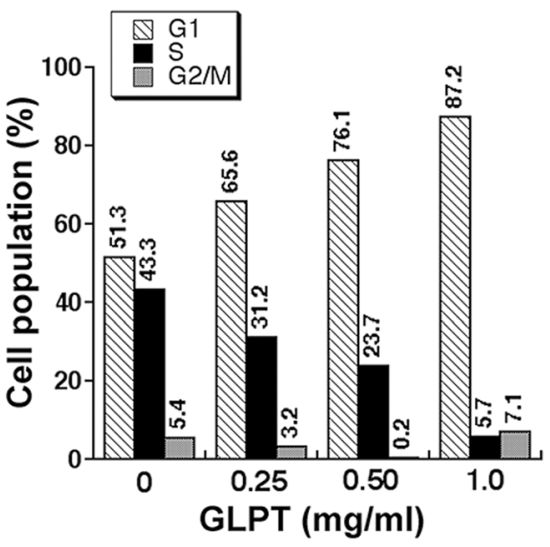

C
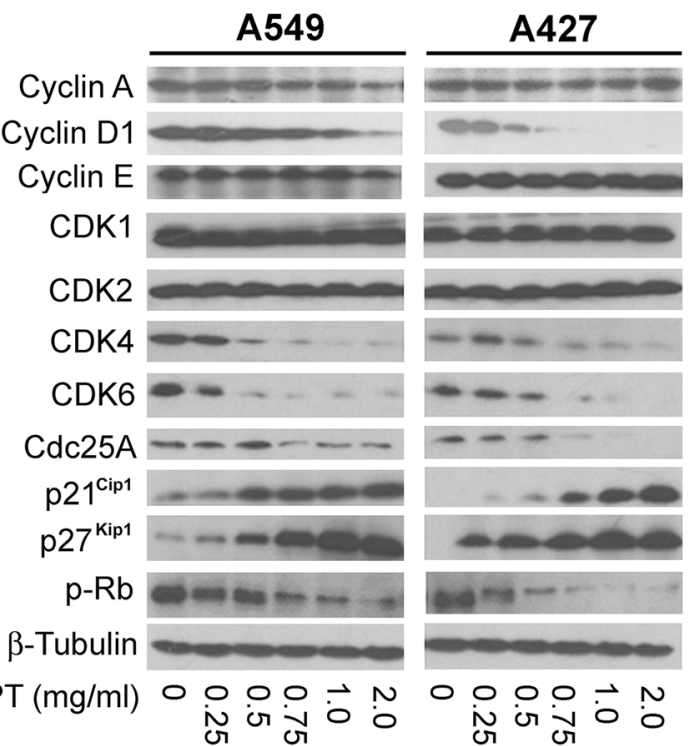

dependently downregulated the protein level of Rheb (Fig. 5b), a direct activator of mTORC $1 .{ }^{39,40}$ In addition, GLPT treatment also activated the AMPK network, as evidenced by increased phosphorylation of AMPKa (T172) and the two substrates of AMPK, TSC2 (S1387) and raptor (S792), in A549 cells (Fig. 5c). Interestingly, ectopic expression of dominant-negative AMPKa 
Fig. 2 GLPT arrests lung cancer cells in the $\mathrm{G}_{1}$ phase of the cell cycle by downregulating the protein levels of cyclin D1, Cdc25A, and CDK4/6 and upregulating the protein levels of p21 ${ }^{\mathrm{Cip} 1}$ and $\mathrm{p} 27^{\mathrm{Kip} 1}$, leading to hypophosphorylation of Rb. a, b A549 cells were treated with GLPT $(0-1 \mathrm{mg} / \mathrm{ml})$ for $24 \mathrm{~h}$ and then subjected to cell cycle analysis. Representative images of the flow cytometry results are presented in (a), and the quantitative data for (a) are shown in $\mathbf{b}$. The bar graphs show the effect of GLPT on the proportion (\%) of A549 cells in the $G_{1}, S$, and $G_{2} / M$ phases of the cell cycle. Similar results were observed in three independent experiments. c A549 and A427 cells were treated with GLPT for $24 \mathrm{~h}$ at the indicated concentrations, and western blotting was then performed with the indicated antibodies. $\beta$-Tubulin served as a loading control

a

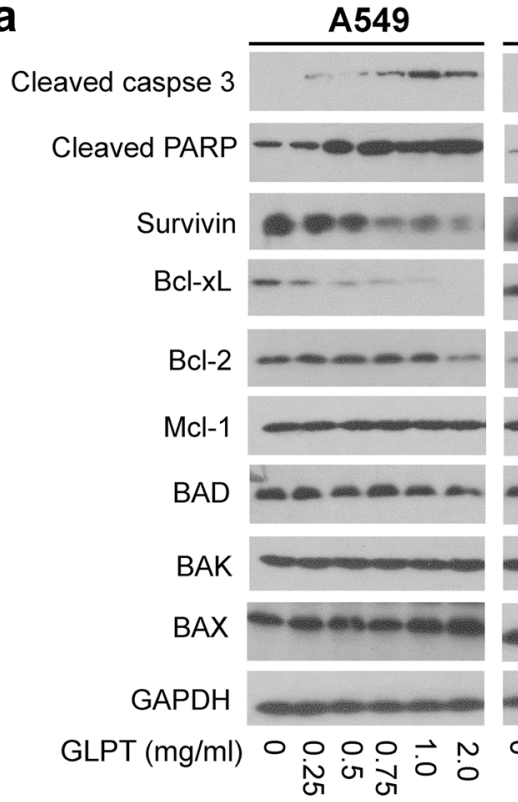

b

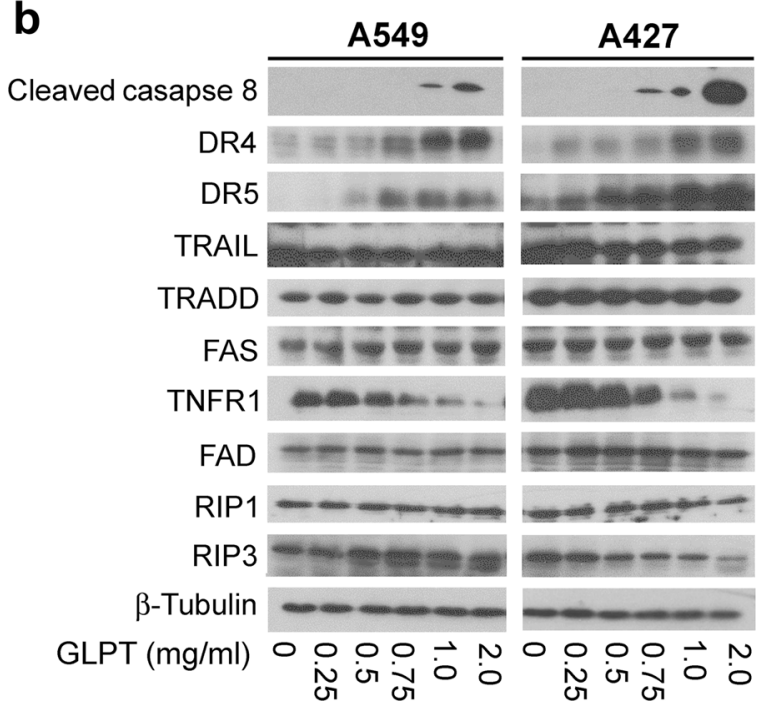

Fig. 3 GLPT downregulates the expression of the antiapoptotic proteins $\mathrm{BCl}-\mathrm{xL}$ and survivin and upregulates the expression of DR4 and DR5, leading to activation of caspase $3 / 8$ and cleavage of PARP. a, b A549 and A427 cells were treated with GLPT for $24 \mathrm{~h}$ at the indicated concentrations, and western blotting was then performed with the indicated antibodies. GAPDH or $\beta$-tubulin served as a loading control

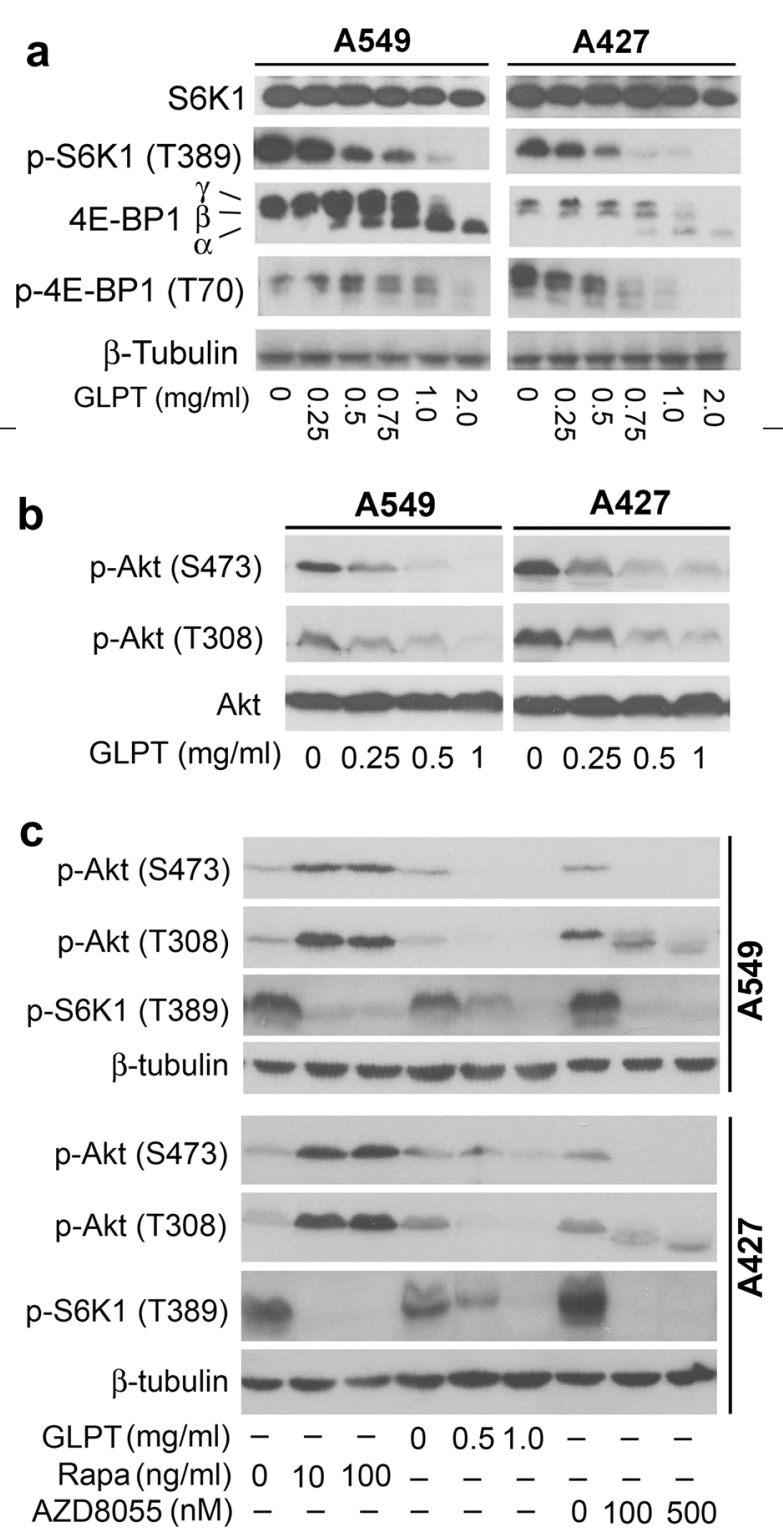

Fig. 4 GLPT inhibits mTORC1/2 signaling pathways. a-c A549 and A427 cells were treated with GLPT for $24 \mathrm{~h}$, rapamycin (Rapa) for $2 \mathrm{~h}$ or AZD8055 for $2 \mathrm{~h}$ at the indicated concentrations, and western blotting was then performed with the indicated antibodies. $\beta$-Tubulin served as a loading control. Similar results were observed in at least three independent experiments

(AMPKa-dn) by infection with Ad-AMPKa-dn partially prevented GLPT from inhibiting mTORC1-mediated S6K1 and 4E-BP1 phosphorylation (Fig. 5d), indicating that GLPT indeed inhibits mTORC1 partly through activation of AMPK. Collectively, these results suggest that GLPT inhibits $\mathrm{mTORC1}$ in part by activating AMPK and inhibiting Rheb in addition to suppressing the IGFRPI3K pathway.

\section{DISCUSSION}

Numerous studies have shown that G. lucidum extracts exert anticancer effects. ${ }^{3-19}$ However, the mechanism behind the anticancer action is not well understood. mTOR plays a pivotal role in controlling cell proliferation and survival. ${ }^{36}$ Here, we found that GLPT, a standardized G. lucidum extract containing both polysaccharides and triterpenes, inhibited cell proliferation and induced cell death and concurrently suppressed mTORC1- 
a

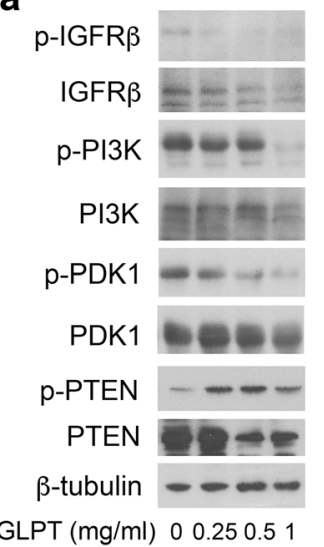

C

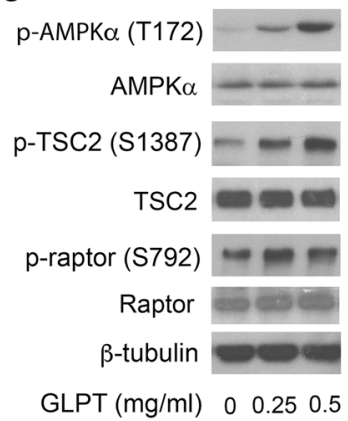

b
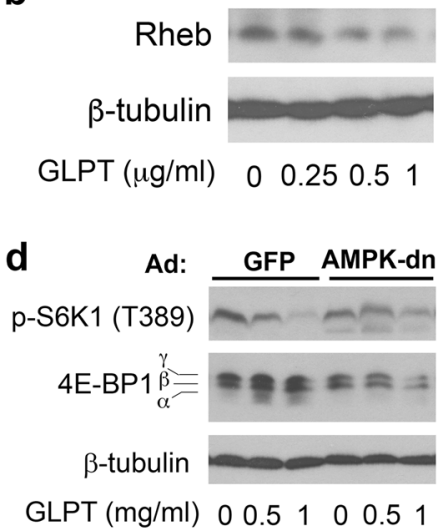

Fig. 5 GLPT-mediated inhibition of mTORC1/2 is associated with activation of AMPK and inhibition of IGFR/PI3K/Rheb. a-d A549 cells (a-c) or A549 cells infected with Ad-AMPK-dn or Ad-GFP (control) (d) were treated with GLPT for $24 \mathrm{~h}$ at the indicated concentrations, and western blotting was then performed with the indicated antibodies. $\beta$-Tubulin served as a loading control

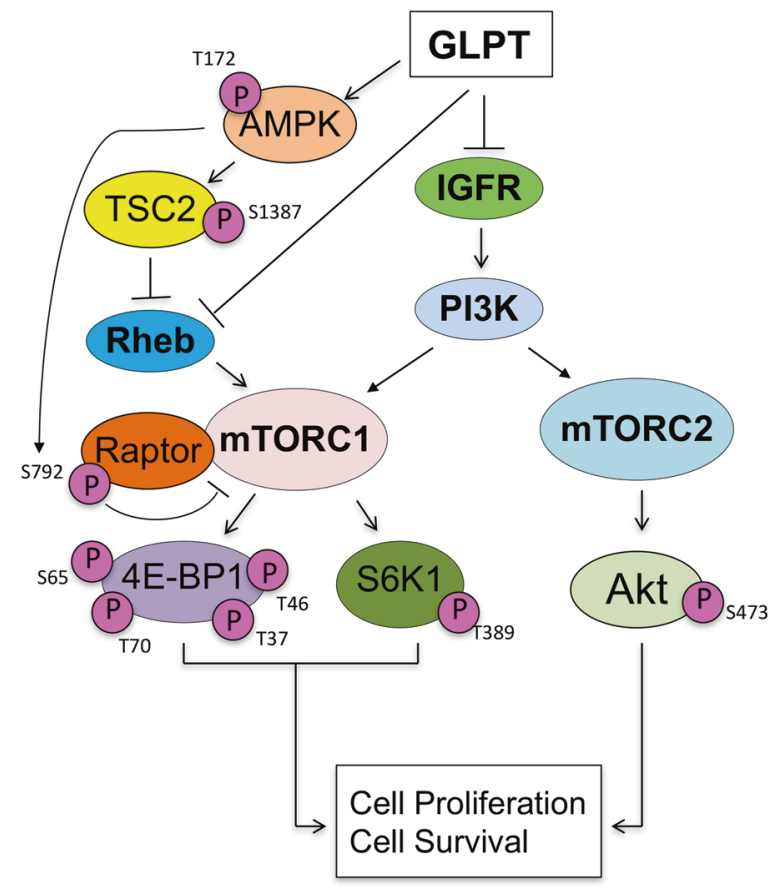

Fig. 6 A diagram showing that GLPT inhibits mTORC1/2-mediated signaling pathways, thereby inhibiting cell proliferation and inducing cell death in tumor cells

mediated phosphorylation of S6K1 and 4E-BP1 and mTORC2mediated phosphorylation of Akt in human lung cancer cells (A549 and A427 cells). Our results are, to some degree, in agreement with some previous findings. ${ }^{14,33,34}$ For instance, treatment with $G$. lucidum polysaccharides inhibits the phosphorylation of 4E-BP1 and ribosomal protein S6 (a substrate of S6K1) in SUM-149 breast cancer cells. ${ }^{34}$ Additionally, treatment with active lipids of $G$. lucidum spores reduces the phosphorylation levels of ERK $1 / 2$ and Akt and increases the phosphorylation level of JNK1/2 in THP-1 leukemia cells. ${ }^{33}$ Together, these findings suggest that inhibition of mTORC1/2 pathways could be one of the main mechanisms underlying the anticancer action of G. lucidum extracts.

In this study, we found that GLPT inhibited the proliferation of A549 and A427 lung cancer cells by inducing cell cycle arrest in $\mathrm{G}_{1}$ phase. This arrest was achieved through reductions in the levels of
$\mathrm{G}_{1} / \mathrm{S}$ proteins, e.g., CDK4, CDK6, cyclin D1, and Cdc25A, and increases in the levels of $\mathrm{p} 27^{\mathrm{Kip} 1}$ and p21 ${ }^{\mathrm{Cip} 1}$ (CDK inhibitors), resulting in hypophosphorylation of $\mathrm{Rb}$ (Fig. 2). In addition, we also noticed that GLPT induced caspase-dependent apoptosis through both the mitochondrial and death receptor pathways. This effect was evidenced by observations that GLPT increased the levels of cleaved caspase 3/8 and cleaved PARP by reducing the levels of antiapoptotic proteins ( $\mathrm{BCl}-\mathrm{xL}$, survivin, and $\mathrm{BCl}-2)$ and increasing the levels of DR4 and DR5 (death receptors) in the cells (Fig. 3). Previous studies on other cell lines have shown that $G$. lucidum extracts exert anticancer effects by downregulating the expression/activity of c-myc, cyclin D1/B1, CDKs, survivin, NF-kB, FAK, small GTPases, MMP-2, and VEGF and upregulating the expression/activity of $\mathrm{p} 27^{\mathrm{Kip} 1}$ and $\mathrm{JNK}^{20-35}$ mTOR is known as a central controller of cell growth/proliferation, motility, and survival. ${ }^{36,42}$ It has been demonstrated that mTOR mediates the expression/activity of c-myc, cyclin D1, CDKs, p27 $7^{\text {Kip1 }}$, VEGF, survivin, JNK, MMP-2, and NF-KB. ${ }^{42}$ This study revealed that GLPT inhibited both mTORC1 and mTORC2 signaling (Fig. 4). In view of our data and that of others, ${ }^{20-35}$ we here tentatively propose that G. lucidum extracts might execute anticancer activity primarily by inhibiting mTOR, indirectly resulting in the up/downregulation of many other signaling molecules. Undoubtedly, further research is needed to confirm this viewpoint.

It is known that rapamycin, the first described allosteric mTOR inhibitor, inhibits mTORC1 but activates mTORC2-mediated phosphorylation of Akt (S473) through a negative feedback mechanism, while AZD8055 (an mTOR kinase inhibitor) inhibits both mTORC1 and mTORC2. ${ }^{36,42}$ This study found that GLPT, similar to AZD8055, inhibited both mTORC1-mediated S6K1 and 4E-BP1 phosphorylation and mTORC2-mediated Akt (S473) phosphorylation (Fig. 4C). However, unlike AZD8055 or rapamycin, GLPT was able to induce AMPKa (T172) phosphorylation and subsequently induce AMPK-mediated TSC2 (S1387)/raptor (S792) phosphorylation (Fig. 5c). Expression of dn-AMPKa partially lessened the inhibitory effect of GLPT on mTORC1 (Fig. 5d), indicating that GLPT inhibits mTORC1 partly via activation of AMPK. In addition, GLPT also reduced the protein level of Rheb (Fig. 5b), a direct activator of mTORC1. ${ }^{39,40}$ Moreover, GLPT was also able to suppress the IGFR-PI3K pathway in the cancer cells (Fig. 5a). Taken together, our data demonstrate that GLPTmediated inhibition of $\mathrm{mTORC} 1 / 2$ is a consequence of inhibition of the IGFR-PI3K pathway, activation of the AMPK-TSC2/raptor network and downregulation of the protein expression of Rheb. Therefore, our findings suggest that GLPT may be a novel mTOR inhibitor. How GLPT activates AMPK and inhibits IGFR/PI3K/Rheb to cause the inhibition of $\mathrm{mTORC} 1 / 2$ remains unclear. Studies have 
shown that increased levels of reactive oxygen species (ROS) or intracellular calcium can modify the structures and functions of cellular proteins, resulting in their inhibition or activation. ${ }^{47}$ It has been described that $G$. lucidum extracts can increase intracellular calcium levels and induce ROS production in MCF-7 breast cancer cells. ${ }^{48}$ Hence, it will be interesting to determine whether GLPT inhibits mTORC $1 / 2$ signaling by increasing the intracellular levels of ROS, calcium or both.

In conclusion, we have demonstrated that GLPT (G. lucidum extracts) inhibited cell proliferation by decreasing the protein levels of CDK4/6, cyclin D1, and Cdc25A; increasing the protein levels of $\mathrm{p} 27^{\mathrm{Kip} 1}$ and $\mathrm{p} 21^{\mathrm{Cip} 1}$; inducing apoptosis by downregulating $\mathrm{BCl}-\mathrm{xL}$, survivin and $\mathrm{Bcl}-2$; and upregulating DR4 and DR5 in human lung cancer cells. Additionally, GLPT not only inhibited mTORC1mediated $\mathrm{S} 6 \mathrm{~K} 1$ and 4E-BP1 phosphorylation but also repressed mTORC2-mediated Akt phosphorylation. This effect was associated with inhibition of the IGFR-PI3K pathway, activation of the AMPKTSC2/raptor network, and downregulation of the protein expression of Rheb (Fig. 6). The results support the notion that inhibition of mTORC1/2 pathways may be one of the major mechanisms underlying the anticancer action of $G$. lucidum extracts.

\section{MATERIALS AND METHODS}

Materials

ReishiMax GLp ${ }^{\mathrm{TM}}$, a standardized G. lucidum extract containing $13.5 \%$ polysaccharides and $6 \%$ triterpenes ${ }^{20}$ here termed GLPT, was purchased from Pharmanex (Provo, UT, USA). The GLPT was dissolved in DMSO (dimethyl sulfoxide) to prepare a fresh $50 \mathrm{mg} /$ $\mathrm{ml}$ stock and then diluted to the necessary concentrations with medium before use. Rapamycin and AZD8055 were purchased from LC Laboratories (Woburn, MA, USA) and ApexBio (Boston, MA, USA), respectively. MEM nonessential amino acids, DMEM (Dulbecco's modified Eagle's medium), and 0.05\% trypsin-EDTA were provided by Mediatech (Manassas, VA, USA). FBS (fetal bovine serum) was obtained from Atlanta Biologicals (Lawrenceville, GA, USA). A CellTiter $96^{\circ}$ Aqueous Non-Radioactive Cell Proliferation Assay Kit was purchased from Promega (Madison, WI, USA). A Cellular DNA Flow Cytometric Analysis Kit and an Annexin V-FITC Apoptosis Detection Kit I were obtained from Roche Diagnostics (Indianapolis, IN, USA) and BD Biosciences (San Jose, CA, USA), respectively. The antibodies used in the study included CDK1, cyclin E, TNFR1, FasL, TRAIL, DR4, DR5, TRADD, FADD, RIP1, RIP3, PI3K p85a/ $/ \gamma$, p-IGFR $\beta$ (Tyr1161), IGFR $\beta$ (Santa Cruz Biotechnology, Santa Cruz, CA, USA), $\beta$-tubulin (Sigma, St Louis, MO, USA), cleaved PARP, cleaved caspase 8 , cleaved caspase $3, \mathrm{p}$ S6K1 (T389), S6K1, p-4E-BP1 (T70), 4E-BP1, p-Akt (S473), p-Akt (T308), Akt, p-PDK1 (S241), PDK1, p-AMPKa (Thr172), p-raptor (S792), p-TSC2 (S1387), p-PTEN (Ser380/Thr382/383), PTEN, p-PI3K p85 (Tyr458)/p55 (Tyr199), Rheb, and GAPDH (Cell Signaling, Danvers, MA, USA) antibodies. All other antibodies and reagents were obtained as previously described. ${ }^{49}$

Cell lines and culture

The human lung adenocarcinoma cell lines A549 and A427 were obtained from the ATCC (American Type Culture Collection) (Manassas, VA, USA). Wild-type mouse embryonic fibroblasts (MEFs) were generously provided by Charles J. Sherr (St. Jude Children's Research Hospital, Memphis, TN, USA). All cells were cultured in DMEM supplemented with 10\% FBS and 1\% MEM nonessential amino acids in a humid incubator $\left(37^{\circ} \mathrm{C}\right.$ and $\left.5 \% \mathrm{CO}_{2}\right)$ and trypsinized with $0.05 \%$ trypsin-EDTA for subculture or experiments.

Analyses of cell viability and proliferation

Cell viability was assessed with trypan blue exclusion assay, and cell proliferation was evaluated with one-solution assay and by cell enumeration, as previously described. ${ }^{49}$
Analyses of cell cycle and apoptosis

Cell cycle and apoptosis analyses were conducted as described previously. ${ }^{49}$ In brief, cells that had been exposed to GLPT at 0-2 $\mathrm{mg} / \mathrm{ml}$ for 24-72 $\mathrm{h}$ were trypsinized and stained with a Cellular DNA Flow Cytometric Analysis Kit (for cell cycle progression) or an Annexin V-FITC Apoptosis Detection Kit I (for apoptosis) and then subjected to flow cytometry using a FACSCalibur flow cytometer (Becton Dickinson, San Jose, CA, USA). Cells treated with DMSO alone served as controls.

Recombinant adenoviruses and infection of cells The recombinant adenoviruses, including Ad-AMPKa-dn encoding c-myc-tagged dominant-negative AMPKa and Ad-GFP encoding GFP (green fluorescence protein), have been described previously. ${ }^{50}$ For experiments, A549 cells were infected with the appropriate adenoviruses for $24 \mathrm{~h}$ at a multiplicity of infection (MOI) of 1. Ad-GFP-infected cells served as controls.

\section{Western blotting}

For western blotting, cells were briefly rinsed with ice-cold PBS (phosphate-buffered saline) and lysed. Then, the cell lysates were separated on an SDS-polyacrylamide gel, transferred to a PVDF membrane, and probed with the indicated antibodies, as described previously. ${ }^{49}$

Statistical analysis

The results are presented as the mean \pm SD (mean value \pm standard deviation). Group variability and interactions were analyzed using one-way ANOVA, and Bonferroni's posttests were used to compare replicate means. $P<0.05$ was considered to indicate significance.

\section{ACKNOWLEDGEMENTS}

We thank Dr. Charles Sherr for generously providing the MEFs. This work was supported by the Scientific and Technological Research Council of Turkey (TUBITAK2219; D. Sohretoglu), the Scientific Research Fund of Hacettepe University (TBI-2015-8365; D. Sohretoglu), the Jiangsu Health International (Regional) Exchange Support Program (C. Zhang), the Jiangsu Provincial Project of Invigorating Health Care through Science, Technology and Education (C. Zhang), the Graduate Student Overseas Study Program of South China Agricultural University (2017LHPY028, J. Luo), the National Institutes of Health (CA115414; S. Huang), the American Cancer Society (RSG-08-135-01-CNE; S. Huang), and the Feist-Weiller Cancer Center at the LSU Health Sciences Center in Shreveport, LA.

\section{AUTHOR CONTRIBUTIONS}

D.S., C.Z., and S.H. conceived the project and designed the study. D.S., C.Z., and J.L. performed the experiments. D.S. C.Z., and S.H. analyzed the data and wrote the manuscript.

\section{ADDITIONAL INFORMATION}

Competing interests: The authors declare no competing interests.

\section{REFERENCES}

1. Lin, Z. B. \& Zhang, H. N. Anti-tumor and immunoregulatory activities of Ganoderma lucidum and its possible mechanisms. Acta Pharmacol. Sin. 25, 1387-1395 (2004).

2. Sohretoglu, D. \& Huang, S. Ganoderma lucidum polysaccharides as an anti-cancer agent. Anticancer Agents Med. Chem 18, 667-674 (2018).

3. Harhaji Trajković, L. M. et al. Anticancer properties of Ganoderma lucidum methanol extracts in vitro and in vivo. Nutr. Cancer. 61, 696-707 (2009).

4. Barbieri, A. et al. Anticancer and anti-inflammatory properties of Ganoderma lucidum extract effects on melanoma and triple-negative breast cancer treatment. Nutrients 9, pii: E210 (2017).

5. Müller, C. I. et al. Ganoderma lucidum causes apoptosis in leukemia, lymphoma and multiple myeloma cells. Leuk. Res. 30, 841-848 (2006).

6. Kim, K. C., Kim, J. S., Son, J. K. \& Kim, I. G. Enhanced induction of mitochondrial damage and apoptosis in human leukemia HL-60 cells by the Ganoderma 
lucidum and Duchesnea chrysantha extracts. Cancer Lett. 246, 210-217 (2007).

7. Sliva, D. et al. Ganoderma lucidum suppresses motility of highly invasive breast and prostate cancer cells. Biochem. Biophys. Res. Commun. 298, 603-612 (2002).

8. Jiang, J., Slivova, V., Valachovicova, T., Harvey, K. \& Sliva, D. Ganoderma lucidum inhibits proliferation and induces apoptosis in human prostate cancer cells PC-3. Int. J. Oncol. 24, 1093-1099 (2004).

9. Hsieh, T. C. \& Wu, J. M. Suppression of proliferation and oxidative stress by extracts of Ganoderma lucidum in the ovarian cancer cell line OVCAR-3. Int. J. Mol. Med. 28, 1065-1069 (2011).

10. Lu, Q. Y. et al. Ganoderma lucidum extracts inhibit growth and induce actin polymerization in bladder cancer cells in vitro. Cancer Lett. 216, 9-20 (2004).

11. Schmidt, M., Polednik, C., Roller, J. \& Hagen, R. Cytotoxicity of herbal extracts used for treatment of prostatic disease on head and neck carcinoma cell lines and non-malignant primary mucosal cells. Oncol. Rep 29, 628-636 (2013).

12. Tang, W., Liu, J. W., Zhao, W. M., Wei, D. Z. \& Zhong, J. J. Ganoderic acid T from Ganoderma lucidum mycelia induces mitochondria mediated apoptosis in lung cancer cells. Life Sci. 80, 205-211 (2006).

13. Sadava, D., Still, D. W., Mudry, R. R. \& Kane, S. E. Effect of Ganoderma on drugsensitive and multidrug-resistant small-cell lung carcinoma cells. Cancer Lett. 277, 182-189 (2009).

14. Chen, Y. et al. Sporoderm-broken spores of Ganoderma lucidum inhibit the growth of lung cancer: Involvement of the Akt/mTOR signaling pathway. Nutr. Cancer 68, 1151-1160 (2016)

15. Lin, S. B., Li, C. H., Lee, S. S. \& Kann, L. S. Triterpene-enriched extracts from Ganoderma lucidum inhibit growth of hepatoma cells via suppressing protein kinase $C$, activating mitogen-activated protein kinases and G2-phase cell cycle arrest. Life Sci. 72, 2381-2390 (2003).

16. Weng, C. J. et al. Inhibitory effects of Ganoderma lucidum on tumorigenesis and metastasis of human hepatoma cells in cells and animal models. J. Agric. Food Chem. 57, 5049-5057 (2009).

17. Jang, K. J. et al. Induction of apoptosis by ethanol extracts of Ganoderma lucidum in human gastric carcinoma cells. J. Acupunct. Meridian Stud. 3, 24-31 (2010).

18. Hong, K. J., Dunn, D. M., Shen, C. L. \& Pence, B. C. Effects of Ganoderma lucidum on apoptotic and anti-inflammatory function in $\mathrm{HT}-29$ human colonic carcinoma cells. Phytother. Res. 18, 768-770 (2004).

19. Jedinak, A., Thyagarajan-Sahu, A., Jiang, J. \& Sliva, D. Ganodermanontriol, a lanostanoid triterpene from Ganoderma lucidum, suppresses growth of colon cancer cells through $\beta$-catenin signaling. Int. J. Oncol. 38, 761-767 (2011).

20. Jiang, J., Slivova, V. \& Sliva, D. Ganoderma lucidum inhibits proliferation of human breast cancer cells by down-regulation of estrogen receptor and NF-KB signaling. Int. J. Oncol. 29, 695-703 (2006).

21. Yang, G., Yang, L., Zhuang, Y., Qian, X. \& Shen, Y. Ganoderma lucidum polysaccharide exerts anti-tumor activity via MAPK pathways in HL-60 acute leukemia cells. J. Recept. Signal Transduct. Res. 36, 6-13 (2016).

22. Hu, H., Ahn, N. S., Yang, X., Lee, Y. S. \& Kang, K. S. Ganoderma lucidum extract induces cell cycle arrest and apoptosis in MCF-7 human breast cancer cell. Int. J. Cancer 102, 250-253 (2002).

23. Dudhgaonkar, S., Thyagarajan, A. \& Sliva, D. Suppression of the inflammatory response by triterpenes isolated from the mushroom Ganoderma lucidum. Int. Immunopharmacol. 9, 1272-12780 (2009).

24. Wang, S. H. et al. Ganoderma lucidum polysaccharides prevent platelet-derived growth factor-stimulated smooth muscle cell proliferation in vitro and neointimal hyperplasia in the endothelial-denuded artery in vivo. J. Cell. Physiol. 227, 3063-3071 (2012).

25. Jiang, J., Grieb, B., Thyagarajan, A. \& Sliva, D. Ganoderic acids suppress growth and invasive behavior of breast cancer cells by modulating AP-1 and NF-KB signaling. Int. J. Mol. Med. 21, 577-584 (2008).

26. $\mathrm{Na}$, K. et al. Anticarcinogenic effects of water extract of sporoderm-broken spores of Ganoderma lucidum on colorectal cancer in vitro and in vivo. Int. J. Oncol 50, 1541-1554 (2017).

27. Stanley, G., Harvey, K., Slivova, V., Jiang, J. \& Sliva, D. Ganoderma lucidum suppresses angiogenesis through the inhibition of secretion of VEGF and TGF- $\beta 1$ from prostate cancer cells. Biochem. Biophys. Res. Commun. 330, 46-52 (2005).

28. Cao, Q. Z. \& Lin, Z. B. Ganoderma lucidum polysaccharides peptide inhibits the growth of vascular endothelial cell and the induction of VEGF in human lung cancer cell. Life Sci. 78, 1457-1463 (2006).

29. Chen, N. H., Liu, J. W. \& Zhong, J. J. Ganoderic acid Me inhibits tumor invasion through down-regulating matrix metalloproteinases $2 / 9$ gene expression. $J$. Pharmacol. Sci. 108, 212-216 (2008).
30. Martínez-Montemayor, M. M., Acevedo, R. R., Otero-Franqui, E., Cubano, L. A. \& Dharmawardhane, S. F. Ganoderma lucidum (Reishi) inhibits cancer cell growth and expression of key molecules in inflammatory breast cancer. Nutr. Cancer $\mathbf{6 3}$, 1085-1094 (2011).

31. Wu, G. S. et al. Ganoderiol A-enriched extract suppresses migration and adhesion of MDA-MB-231 cells by inhibiting FAK-SRC-paxillin cascade pathway. PLOS ONE. 8, e76620 (2013).

32. Jiang, J., Slivova, V., Harvey, K., Valachovicova, T. \& Sliva, D. Ganoderma lucidum suppresses growth of breast cancer cells through the inhibition of Akt/NF-KB signaling. Nutr. Cancer 49, 209-216 (2004).

33. Wang, J. H., Zhou, Y. J., Zhang, M., Kan, L. \& He, P. Active lipids of Ganoderma lucidum spores-induced apoptosis in human leukemia THP-1 cells via MAPK and PI3K pathways. J. Ethnopharmacol. 139, 582-589 (2012).

34. Suarez-Arroyo, I. J. et al. Anti-tumor effects of Ganoderma lucidum (reishi) in inflammatory breast cancer in in vivo and in vitro models. PLoS ONE. 8, e57431 (2013).

35. Li, X. et al. Ergosterol peroxide activates Foxo3-mediated cell death signaling by inhibiting AKT and c-Myc in human hepatocellular carcinoma cells. Oncotarget. 7, 33948-33959 (2016).

36. Mossmann, D., Park, S. \& Hall, M. N. mTOR signalling and cellular metabolism are mutual determinants in cancer. Nat. Rev. Cancer 18, 744-757 (2018).

37. Berman, A. Y. et al. ERRa regulates the growth of triple-negative breast cancer cells via S6K1-dependent mechanism. Signal Transduct. Target. Ther. 2, pii: e17035 (2017).

38. Inoki, K., Zhu, T. \& Guan, K. L. TSC2 mediates cellular energy response to control cell growth and survival. Cell 115, 577-590 (2003).

39. Tee, A. R., Manning, B. D., Roux, P. P., Cantley, L. C. \& Blenis, J. Tuberous sclerosis complex gene products, Tuberin and Hamartin, control mTOR signaling by acting as a GTPase-activating protein complex toward Rheb. Curr. Biol. 13, 1259-1268 (2003).

40. Inoki, K., Li, Y., Xu, T. \& Guan, K. L. Rheb GTPase is a direct target of TSC2 GAP activity and regulates mTOR signaling. Genes Dev. 17, 1829-1834 (2003).

41. Gwinn, D. M. et al. AMPK phosphorylation of raptor mediates a metabolic checkpoint. Mol. Cell 30, 214-226 (2008).

42. Zhou, H. \& Huang, S. Role of mTOR signaling in tumor cell motility, invasion and metastasis. Curr. Protein Pept. Sci. 12, 30-42 (2011).

43. Malumbres, M. \& Barbacid, M. Cell cycle, CDKs and cancer: a changing paradigm. Nat. Rev. Cancer 9, 153-166 (2009).

44. Fuchs, Y. \& Steller, H. Live to die another way: modes of programmed cell death and the signals emanating from dying cells. Nat. Rev. Mol. Cell. Biol. 16, 329-344 (2015).

45. Tummers, B. \& Green, D. R. Caspase-8: regulating life and death. Immunol. Rev. 277, 76-89 (2017)

46. Yuan, J., Najafov, A. \& Py, B. F. Roles of caspases in necrotic cell death. Cell. 167, 1693-1704 (2016).

47. Görlach, A., Bertram, K., Hudecova, S. \& Krizanova, O. Calcium and ROS: a mutual interplay. Redox Biol 6, 260-271 (2015).

48. Kim, T. H. et al. Induction of apoptosis in MCF-7 human breast cancer cells by Khz (fusion of Ganoderma lucidum and Polyporus umbellatus mycelium). Mol. Med. Rep 13, 1243-1249 (2015).

49. Barzegar, M. et al. SKLB188 inhibits the growth of head and neck squamous cell carcinoma by suppressing EGFR signalling. Br. J. Cancer 117, 1154-1163 (2017).

50. Zhou, $\mathrm{H}$. et al. Ciclopirox olamine inhibits mTORC1 signaling by activation of AMPK. Biochem. Pharmacol. 116, 39-50 (2016).

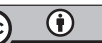

Open Access This article is licensed under a Creative Commons Attribution 4.0 International License, which permits use, sharing, adaptation, distribution and reproduction in any medium or format, as long as you give appropriate credit to the original author(s) and the source, provide a link to the Creative Commons license, and indicate if changes were made. The images or other third party material in this article are included in the article's Creative Commons license, unless indicated otherwise in a credit line to the material. If material is not included in the article's Creative Commons license and your intended use is not permitted by statutory regulation or exceeds the permitted use, you will need to obtain permission directly from the copyright holder. To view a copy of this license, visit http://creativecommons. org/licenses/by/4.0/.

(c) The Author(s) 2019 